\title{
Éc(h)opoétiques : chanter, écouter, éprouver les
} lieux

\section{Mélanie Bourlet, Marie Lorin et Katell Morand}

\section{Q OpenEdition}

1 Journals

Édition électronique

URL : https://journals.openedition.org/clo/8289

DOI : $10.4000 /$ clo.8289

ISSN : 2266-1816

Éditeur

INALCO

\section{Édition imprimée}

Date de publication : 30 juin 2020

Pagination : $9-20$

ISBN : 978-2-85831-392-1

ISSN : 0396-891X

\section{Référence électronique}

Mélanie Bourlet, Marie Lorin et Katell Morand, "Éc(h)opoétiques : chanter, écouter, éprouver les lieux », Cahiers de littérature orale [En ligne], 87 | 2020, mis en ligne le 16 septembre 2021, consulté le 09 novembre 2022. URL : http://journals.openedition.org/clo/8289; DOI : https://doi.org/10.4000/clo. 8289

\section{(c) (7) (8)}

Creative Commons - Attribution - Pas d'Utilisation Commerciale 4.0 International - CC BY-NC 4.0

https://creativecommons.org/licenses/by-nc/4.0/ 


\title{
Éc(h)opoétiques : chanter, écouter, éprouver les lieux
}

Mélanie Bourlet

Marie LORIN

Katell Morand

\begin{abstract}
« À travers le chant, un auditeur Kaluli est suspendu dans les lieux. Il passe à côté et à travers eux, fait un grand tour intérieur dans le micro espace-temps intériorisé de l'écoute. » (Feld, 1996, traduit dans ce numéro)
\end{abstract}

"Relaxing at the creek" : c'est par ce titre d'une grande simplicité qu'est introduite la voix d'Ulahi dans le disque Voices of the Rainforest de Steven Feld, portrait sonore d'une journée dans la vie des Kaluli de Papouasie-Nouvelle-Guinée (Feld, 1991). Ulahi chante au ruisseau et sa voix se superpose aux textures denses des sons de la forêt tropicale. Égrenant des noms de lieux, elle invite l'auditeur, tel un oiseau triste survolant la canopée, à un voyage le long des cours d'eau, s'arrêtant près des arbres solitaires et des jardins cultivés, soulignant la mélancolie qui émane des habitats désertés. Semblable au mouvement de l'eau qui entoure la chanteuse, cette composition est « comme une cascade dans [la] tête ».

Des années 1970 au milieu des années 1990, l'anthropologue Steven Feld a consacré ses travaux à la langue et aux musiques des Kaluli. Son premier livre, Sound and Sentiment. Birds, Weeping, Poetics and Song in Kaluli Expression, publié en 1982, prend tout autant les allures d'un traité d'ornithologie que celles d'une recherche sur la musique. On y découvre l'importance des oiseaux, 
présences dans la forêt, métaphores centrales de l'attachement et de l'abandon, et dont les chants et les pleurs se font l'écho. Mais c'est dans le texte "Waterfalls of Song”, paru en 1996 dans Senses of Places, un ouvrage co-édité avec Keith Basso et qui fit date dans l'histoire de l'anthropologie ${ }^{1}$, que sont interrogés les rapports de la poétique et de la musique avec la sensorialité des lieux. Cartographiant leurs histoires, les faisant « résonner émotionnellement avec la conscience de soi personnelle, biographique et historique », les chanteurs Kaluli développent ce que Steven Feld nomme une acoustémologie, autrement dit une épistémologie par le son, condition de la connaissance et de la fabrication du lieu.

Malgré son influence durable en anthropologie et en ethnomusicologie ${ }^{2}$, ce texte - exigeant dans sa langue même, où métaphores et jeux de mots répondent aux retournements de sens appréciés des Kaluli - reste peu connu des spécialistes de littérature. L'objectif de ce numéro, en l'introduisant grâce à la belle traduction de Lila Lakehal à un public francophone et littéraire ${ }^{3}$, est de mesurer ses échos vingt ans après sa publication et alors que Voices of the Rainforest vient d'être réédité sous forme de film ${ }^{4}$.

Au moment où se développe un courant «écopoétique » dans les études littéraires, il s'agit de prendre la mesure de ce que représente l'oralité dans l'expérience vécue et située des environnements : en suivant le fil des engagements sensoriels, corporels et émotionnels qui sont en jeu dans la performance ; en prêtant une attention conjointe à l'écoute des lieux et aux formes sonores de créativité ; et enfin en étudiant les attachements qui sont produits, transmis et parfois profondément transformés.

\section{De l'écopoétique à l'éc(h)opoétique}

Dans un contexte de changements climatiques et d'attention de plus en plus forte aux risques environnementaux qui menacent l'équilibre écologique de

1. L'article qu'y propose Keith Basso, "Wisdom Sits in Places", fut ensuite repris dans l'ouvrage du même nom récemment traduit en Français (2016).

2. Cf. Feld \& Brenneis, 2004 ; Feld \& Boudreault-Fournier, 2019.

3. Ce numéro fait suite à la journée d'études organisée le 29 mai 2019, en collaboration avec le Llacan et le CREM, à l'Inalco. Le programme de ces journées est consultable sur http://www.inalco.fr/actualite/journee-etudes-ec-h-opoetiques-chanter-ecouter-eprouverlieux-29-mai-2019. Il est possible de visionner cette journée sur youtube : https://www. youtube.com/watch? $\mathrm{v}=77 \mathrm{cexXllaVs}$.

4. Feld, Leonard \& Richards, 2019. 
la planète, les sciences humaines s'emparent de domaines qui jusqu'il y a peu semblaient réservés aux sciences dites dures. Ainsi en est-il de l'actualité mondiale autour de la propagation de la COVID-19. Révélatrice à bien des égards de liens d'interdépendances tout autant que de la fragilisation de ces derniers, celle-ci met en lumière la nécessité de repenser nos rapports au vivant et aux lieux. Nous traversons, comme le souligne le géographe Michel Lussault ${ }^{5}$, une crise d' « habitabilité » planétaire qui pose plus que jamais la question de la cohabitation et fait des vulnérabilités collectives et individuelles autant d'enjeux sociétaux. Au cœur des préoccupations se retrouve la question du soin à accorder aux cultures, aux écosystèmes, aux idées ou encore aux individus. La question écologique s'étend bien au-delà des crises environnementales ${ }^{6}$ : elle pointe surtout la nécessité de prêter attention à la diversité des modes de relation et de décentrer la place de l'être humain. Comme l'a écrit Baptiste Morizot (2020), « la crise écologique systémique qui est la nôtre est aussi une crise de la sensibilité au vivant. J'entends par là l'appauvrissement des mots, des capacités à percevoir, des émotions et des relations que nous pouvons tisser avec le monde vivant $\gg$. L'écologie n'est donc pas seulement un problème de chiffres ou de mesure, mais aussi une question de récits que les sociétés élaborent d'elles-mêmes et de leur environnement : elle devient peu à peu un enjeu majeur de poétique relationnelle, aux vivants et aux lieux; en d'autres termes, une « écologie du sensible », celle-là même que Tim Ingold (2013) associe à une « poétique de l'habiter ».

L'essor de l'écopoétique dans le corpus théorique francophone reprend en partie les jalons posés par l'écocritique américaine, concentrée essentiellement sur la perspective éthique et politique de l'étude des rapports entre littérature

5. Michel Lussault s'est interrogé, seul ou avec d'autres, sur la question de l'« habitabilité » à travers plusieurs de ses publications, (cf Lussault, PaQuot \& Younès, 2007), définissant le terme « habiter » comme le « rapport que l'homme entretient avec les lieux de son existence, mais aussi à la relation, sans cesse renouvelée, qu'il établit avec l'écoumène, cette demeure terrestre de l'être ». Dans son dernier ouvrage, Chroniques de géo' virale (2020), il interroge notamment ce concept d'habitabilité, et plus largement celui de l'espace, à partir de la situation sanitaire liée à la COVID-19 et du confinement, opportunités selon lui pour penser le monde, qui remet au cœur des débats socio-politiques la nécessité du lieu, de la cohabitation et du soin à apporter aux vulnérabilités.

6. Nous reprendrons ici la définition du terme « écologie » (étymologiquement « discours sur l'habitat », éco < oikos : maison, habitat et logie < logos : discours) donnée par Haeckel (1866) : « La science des relations des organismes avec le monde environnant, c'est-à-dire, dans un sens large, la science des conditions d'existence. » 
et environnement ${ }^{7}$. L'écopoétique propose cependant d'insister plus fortement sur les formes d'écriture que de tels engagements supposent. En nous appuyant sur des propositions issues de l'anthropologie du sonore, nous proposons ici une éc(h)opoétique dont le « $\mathrm{h} »$ appelle à des aller-retours entre texte et son, propices à une écoute de la sensorialité des lieux. Les articles de ce numéro interrogent chacun à leur façon des poétiques orales qui mettent en question ou décrivent des relations que les vivants - humains ou non - établissent entre eux et avec l'environnement et montrent une diversité d'approches écologiques, alors même qu'un seul modèle productiviste et extractiviste semble s'être imposé à tous. Interroger ces oralités, jusqu'ici peu prises en compte dans les études littéraires, permet de comprendre comment, au-delà des crises actuelles, ces formes langagières et sonores sont des lieux d'élaboration de manières d'être au monde.

Ce numéro allie donc une triple transversalité. Transversalité disciplinaire d'abord qui associe ethnomusicologues, littéraires et anthropologues ; transversalité formelle ensuite, allant de la poésie chantée ou déclamée aux récits de vie, aux conversations, et même aux comptes rendus écrits d'expériences sonores ; transversalité géographique et linguistique enfin puisque se côtoient des voix aborigènes d'Australie, peules du Mâcina, sámi d'Europe septentrionale, ou encore celles de chasseurs du Jura. C'est à partir de cette diversité de cas que ce numéro explore les façons dont l'expérience des lieux et la création verbale, en particulier orale et chantée, se nourrissent et se façonnent mutuellement. Il entre ainsi en résonance avec les questionnements et motifs présents dans le texte de Steven Feld.

\section{Marche et parcours sensibles}

Plusieurs articles de ce numéro présentent des parcours, des itinéraires, des traversées. Ils interrogent les façons dont le chant et les mots proposent des représentations mentales de lieux dans lesquels la voix humaine n'est qu'un élément d'un vaste écosystème sonore. Le fil de la voix dans sa dimension éphémère permet une mise en réseau de différentes sources sonores qui sont autant de modes de connaissances des lieux. Le relief, par exemple, n'est pas seulement l'objet de la connaissance, mais sa condition même. Ainsi chez les Kaluli, comme l'écrit Steven Feld, les « cuisses » et « flancs » du paysage sont sources d'échos

7. Sur l'écocritique et l'écopoétique, voir la bibliographie du site ZoneZadir, (collectif « pour une écopoétique transculturelle ») : https://zonezadir.hypotheses.org/bibliographie. 
et de réverbérations propres, comme celles de l'eau sur les pentes douces ou accidentées : sons de chute ou d'aspersion, tourbillonnant dans les bassins ou éclaboussant les berges, et dont les onomatopées ponctuent les textes chantés. L'article de Marie Lorin s'inscrit dans cette perspective. Il montre que l'écoute du fleuve est au centre des chants des pêcheurs mais aussi de leurs connaissances du fleuve Sénégal. L'écoulement de l'eau, motif central des cascades de chants décrits par Feld est également un motif central dans le genre du Pékâne. La crue, les maelströms, les courants sont à la fois des motifs rythmiques et poétiques, le signe d'une maîtrise de l'environnement fluvial mais aussi un mode de mise en relation entre humains et formes de vie habitant le fleuve. Le passage d'un lieu à un autre permet le déroulement du poème tout en se faisant l'écho de la marche au bord de l'eau du chanteur.

La mise en mouvement du corps par la marche est aussi un engagement sensible qui permet des attentions différenciées à des strates sonores juxtaposées ou successives, présentes autour de soi. La pensée se saisit de ces variations sensorielles dans le travail de composition poétique et sonore. Ainsi, dans l'article de Giordano Marmone consacré aux pasteurs samburu du Kenya, le chant des jeunes circoncis de retour de raid permet de domestiquer un territoire appartenant à leurs ennemis. Leur expérience d'un environnement parcouru dans la hâte et la tension de la fuite est réactualisée lors des performances par les mouvements de marche collectifs qui accompagnent les récits chantés. Au sein de cette société à classes d'âges, ces chants singularisent chaque génération successive et offrent des moyens de penser les transformations contemporaines des relations et des espaces de vie.

Dans tous ces articles, les compétences des chanteurs et poètes sont étroitement liées à leurs savoirs environnementaux. De la même façon que les chanteurs Kaluli sont des ornithologues, les pêcheurs du fleuve Sénégal sont de fins connaisseurs de la faune et de la flore, tandis que les poètes peuls du Mâcina, dont les textes sont analysés dans l'article de Christiane Seydou, témoignent de leur talent de topographes des chemins de transhumance. Cette extrême précision des savoirs a donc poussé certains auteurs de ce numéro à un travail d'enquête ethnobotanique ou ethnozoologique nécessaire à la compréhension des usages, des métaphores et des imaginaires associés aux animaux, aux végétaux et aux lieux dont les textes se font parfois les « conservatoires » lexicaux, pour reprendre l'expression de Christiane Seydou. Mais les relations ne sont pas aussi univoques qu'elles pourraient le sembler de premier abord. Dans son article, Christiane Seydou montre que le vocabulaire mobilisé l'est tout autant, sinon plus, pour ses qualités phoniques que pour sa référentialité : les mots sont utilisés dans un jeu virtuose comme autant de matériaux sonores propices à des agencements 
poétiques et rythmiques originaux. À travers la notion de « continuum sonore », par exemple, et grâce à des analyses stylistiques très fines, elle montre comment la forme poétique fait écho de façon synesthésique à la réalité de la transhumance au fil des saisons et au défilé continu et haut en couleurs des bovins.

\section{Points d'écoutes}

Cette entrée par le son, qui guide l'attention et la manière dont se construit un savoir et une forme poétique, est illustrée dans ce numéro par plusieurs contributeur-rices. Les articles témoignent d'un glissement d'une perspective située sur le « point de vue » vers ce qui serait « un point d'oreille » (earpoint) ou « point d'audition » (dans la traduction de Lila Lakehal). Originellement proposé par Carpenter (1960) dans son analyse de l'expérience de l'espace des Inuits, ce concept opère un mouvement parallèle à celui de soundscape du compositeur Murray Schafer (1977), en cherchant à déplacer vers l'ouie un rapport à l'environnement où la vue aurait acquis une importance prééminente dans la pensée occidentale ${ }^{8}$.

Comment interpréter ce que l'on entend ou que d'autres nous disent entendre, comment le rationaliser pour lui donner sens ? Le document proposé par Jean-Marie Privat expose les troubles du curé d'Ansacq (en Beauvaisis), qui fait appel en 1730 au discernement des lecteurs du Mercure de France pour comprendre un phénomène sonore nocturne, violent et aérien - « un charivari épouvantable » - entendu dans la campagne par un groupe de villageois. Après avoir relaté ses expériences infructueuses pour tenter de reproduire ce « prodige », le curé d'Ansacq propose le terme d'acousmate pour désigner ce type de phénomène inquiétant fait de voix et de sons d'instruments, terme qui sera repris très vite dans les dictionnaires modernes. Dans sa volonté de comprendre une perception qui lui échappe, il nous invite donc à nous interroger sur le sens des « distorsions » d'écoute et sur la manière de les appréhender méthodologiquement. Ce qui s'ouvre alors en filigrane à travers ces expériences sonores, c'est la possibilité de mettre en lumière la manière dont les sons inquiètent, révèlent, parlent, apaisent, dévoilent, ouvrent à un monde et ancrent

8. Désignant tout autant l'environnement acoustique, son étude, et la composition (le tuning ou l'accordage) que chacun en fait, soundscape a connu un grand succès et a participé au développement des sound studies et autres études des « paysages sensoriels », cf. Candau \& Le Gonidec, 2013. 
la conscience de soi et la mémoire individuelle et collective sans laquelle, comme le montre Steven Feld, le lieu ne saurait s'incorporer.

À partir de la correspondance d'un pasteur anglais du XVIII ${ }^{\mathrm{e}}$ siècle et de deux « vignettes ethnographiques » (France et Mongolie), Laurent Legrain et Marie Balthazar s'appuient, quant à eux, sur le concept d'acoustémologie pour développer la notion d'《empreinte sonore ». Elle leur permet de mettre en évidence les séries de petites opérations qui sous-tendent les processus d'écoute, depuis l'étonnement initial jusqu'au souvenir : celles du pasteur qui teste l'écho dans sa campagne de vers de Virgile, des chasseurs qui se répartissent des points d'écoute du brame du cerf ou encore du jeune homme d'Ulaanbaatar qui retrouve dans un frottement de tiges son territoire natal. Il s'agit de se placer, d'expérimenter, de mettre en mots et de partager. Tout ce qui est « fait avec et par » les sons façonne ainsi une empreinte dont la forme s'élabore dans l'échange et s'inscrit dans les parcours individuels.

De son côté, Stéphane Aubinet montre qu'il se joue, dans la pratique du yoik, ces courtes mélodies chantées par les Sami d'Europe septentrionale, des transformations individuelles de la perception qu'il nomme enchantements, et qui sont potentiellement partagées par la mise en mots. Il illustre ces processus créatifs, nés d'expérimentations singulières, par deux rencontres. La première, capturée sur vidéo et racontée dans un article du journal en ligne Nordlys en 2014, est celle d'un chanteur avec des élans que le yoik de l'ours aurait fait fuir ; la seconde, retracée par un poème et une peinture, est celle du vent se faufilant à travers les forêts et les montagnes, et dont le souffle révèle des nuances ou «variations » de l'environnement : un monde de possibles, de souvenirs et d'affects.

L'anthropologue Barbara Glowczewski, la linguiste Mary Laughren et le doyen warlpiri Jerry Jangala Patrick présentent la transcription, la traduction et un commentaire d'une songline Warlpiri composée collectivement dans les années 1960 après un mois de visions en rêve de Janjiya Liddy Nakamarra Herbert, traumatisée par l'incendie de ses tablettes sacrées. En 1984, lorsque Barbara Glowczewski a enregistré le rituel du Feu chanté par des hommes et des femmes, la rêveuse lui a expliqué qu'il a permis aux Warlpiri de légitimer leur sédentarisation forcée dans la réserve de Lajamanu, sur les terres de leurs voisins au nord du désert central d'Australie. S'esquisse ici la faculté d'actualisation du chant par un ré-agencement des histoires individuelles et collectives, en d'autres 
termes la possibilité de restaurer un lien devenu traumatique pour investir un nouveau territoire, à travers un processus de création et d'incorporation ${ }^{9}$.

Transversale à tous les articles du numéro est donc la question des formes d'attachement des humains avec leur environnement : animaux, plantes, rochers, vent, cours d'eau, etc. Cette question ouvre une réflexion sur les échelles de ces attachements, leurs dynamiques et les cohabitations parfois conflictuelles et en négociation sur un même territoire.

\section{Ouvertures}

Ces contributions nous invitent également à élargir le champ des investigations de terrain et à mieux saisir les liens entre parcours de vie, attachements aux lieux et créativité orale, ainsi que la manière dont cette créativité s'adapte en continu aux transformations des cadres de vie.

Des perspectives de recherche interdisciplinaires se dessinent, ouvrant la poétique à des questions à l'intersection des sound studies, de la géographie, de l'anthropologie, de la linguistique ou encore de l'histoire. Très concrètement, il s'agit de réfléchir aux conditions de la production des données, à leur mise en forme pour l'analyse et à leur représentation pour un public large. Des prises de son ou encore le dispositif de la promenade sensorielle, tel qu'il est déployé dans un cadre pédagogique ${ }^{10}$, permettent d'éprouver et d'expérimenter des écoutes situées et en mouvement le long d'itinéraires. Par ailleurs, comme plusieurs travaux récents le montrent ${ }^{11}$, des expérimentations cartographiques permettent la représentation de ce type de données sensibles tout en proposant d'autres modes d'organisation des savoirs et des outils d'exploration des imaginaires accessibles au plus grand nombre.

9. Signalons que la mise en tension du corps par le son est utilisée en psychophysique pour appréhender les expériences de stress post-traumatiques associées à des lieux, à l'instar de l'attentat perpétré au Bataclan à Paris le 13 novembre 2015. Il s'agit de comprendre la résonance qu'un lieu peut avoir sur le corps et ainsi, par le biais d'une approche transdisciplinaire associant notamment des musicothérapeuthes, de restaurer par le son le lien au lieu, et donc de corriger le rapport angoissant au monde qui s'est noué dans l'altération du sonore. Cf. le documentaire sonore L'Echo du Bataclan du 10 novembre 2020 de Jérôme Leroux (Arte Audio) : https://www.arteradio.com/ son/61664912/1_echo_du_bataclan.

10. Cf. Gélard, Gosselain \& Legrain, 2016 ; Bouvet, 2019.

11. Cf. Arènes, Grégoire \& AÏT-Touati, 2019. 
Le travail consacré par Steven Feld depuis les années 1990 à des formes expérimentales de représentation sonore ou audiovisuelle de l'écoute des lieux ouvre des perspectives de recherche stimulantes afin de recréer la dimension immersive et dynamique de ces expériences. En témoigne son dernier film, Voices of the rainforest, version en format cinématique 7.1 de son disque de 1991 dont les images ont été tournées en 2018. Dans l'extrait qui accompagne le numéro ${ }^{12}$, on suit ainsi les pas d'Ulahi dans ses trajectoires chantées à travers la forêt tropicale grâce à la division de l'écran en plans représentant son cheminement par points d'écoute : une eau qui s'écoule, un oiseau survolant la canopée, ou le cochon qu'elle appelle. Après des décennies de changements écologiques et sociaux qui ont contribué à la quasi disparition de certains genres poétiques, Steven Feld relève d'ailleurs, dans l'entretien qu'il nous a accordé, un regain d'intérêt chez les plus jeunes pour le savoir sur la forêt tropicale, à présent personnifié par Ulahi et son chant.

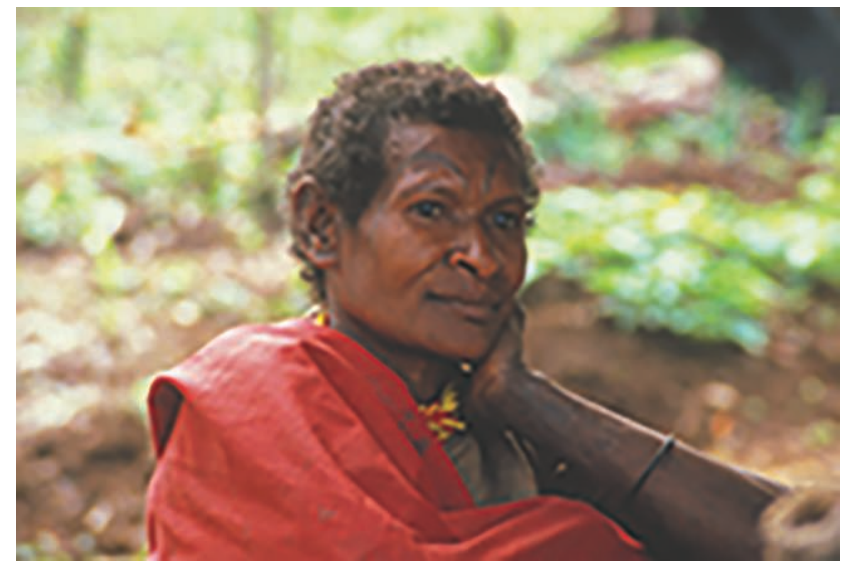

Figure 1

Ulahi écoute son propre enregistrement.

Photographie (C) Steven Feld.

L'imagination et la capacité à se reconnecter aux lieux de vie constituent autant d'horizons d'espoir face au changement climatique et à la transformation des habitats. En d'autres termes, il s'agirait de poursuivre une réflexion sur la

12. L'extrait, dans sa version sous-titrée en français, est disponible sur Internet : https:// vimeo.com/489204199. 
manière dont l'oralité constitue un mode de « résilience écologique » (cf. Sinaï et al., 2017), où l'écoute est un acte d'attention façonné par les liens affectifs entretenus entre milieux et êtres vivants ${ }^{13}$. Défendre les cultures orales, la force du chant et de la littérature, n'est-ce pas aussi défendre la joie créative de faire partie d'un vaste monde tissé en son sein d'une multitude de liens d'interdépendance, et donc d'en défendre l'habitabilité ?

« Bref, s'arrêter, écouter, écouter encore : ici, maintenant, se passe et se crée quelque chose d'important » nous dit Vinciane Desprets dans son dernier ouvrage Habiter en oiseau (2019). Et de poursuivre, dans une réflexion sur les oiseaux qui fait écho aux questionnements de ce numéro : « C'est cela également que pourrait signifier le fait d'inscrire notre époque, comme le propose Donna Haraway, sous le signe du "Phonocène" ${ }^{14}$. C'est ne pas oublier que si la terre gronde et grince, elle chante également. C'est ne pas oublier non plus que ces chants sont en train de disparaître, mais qu'ils disparaîtront d'autant plus si on n'y prête pas attention. Et que disparaîtront avec eux de multiples manières d'habiter la terre, des intentions de vie, des compositions, des partitions mélodiques, des appropriations délicates, des manières d'être et des importances. Tout ce qui fait des territoires et tout ce que font des territoires animés, rythmés, vécus, aimés. Habités. Vivre notre époque en la nommant "Phonocène", c'est apprendre à prêter attention au silence qu'un chant de merle peut faire exister, c'est vivre dans des territoires chantés, mais c'est également ne pas oublier que le silence pourrait s'imposer. Et ce que nous risquons bien de perdre également, faute d'attention, ce sera le courage chanté des oiseaux ${ }^{15}$. »

13. D'où l'intérêt d'un néologisme comme celui d' «auralité », qui insiste sur la prise en compte de l'auditeur et met l'accent sur « la matérialité du corps parlant (la voix) et du corps écoutant (l'oreille) », PRIVAT, 2019.

14. Sur le choix du terme « phonocène » (plutôt qu'anthropocène, plantationocène, etc), Vinciane Despret s'explique dans un entretien en ligne http://www.mouvement.net/ teteatete/entretiens/vinciane-despret : «J'aime beaucoup l'idée que nous sommes à l'ère du Phonocène, c'est-à-dire à "l'ère des sons de la Terre", parce que cela engage un régime d'attention particulier au vivant tel qu'il est : encore vivant, et pas seulement en voie de disparition. » 


\section{Références}

Arènes Alexandra, Grégoire Axelle \& Aït-Touati Frédérique, 2019, Terra Forma. Manuel de cartographies potentielles, Éditions B42, Paris, 192 p.

BAsso Keith, 2016, L'Eau se mêle à la boue dans un bassin à ciel ouvert: paysage et langage chez les Apaches occidentaux, Zones sensibles, Paris, 196 p.

Candau Joël \& Le Gonidec Marie-Barbara, 2013, Paysages sensoriels : essai d'anthropologie de la construction et de la perception de l'environnement sonore, CTHS, Paris, 239 p.

Carpenter Edmund, 1960, "Acoustic Space" in Carpenter Edmund \& McLuhan Marshall (eds.), Explorations in Communication: An Anthology, Beacon Press, Boston, pp. 65-70.

Despret Vinciane, 2019, Habiter en oiseau, Actes Sud, Arles, 224 p.

Feld Steven, 1982, Sound and Sentiment. Birds, Weeping, Poetics and Songs in Kaluli Expression, University of Pennsylvania Press, Philadelphia, 297 p.

Feld Steven, 1991, Voices of the Rainforest: A Day in the Life of the Kaluli People, CD. The World series, Mickey Hart, ed. Rykodisc, Boston.

Feld Steven, 1996, "Waterfalls of Song: An Acoustemology of Place Resounding in Bosavi, New Guinea" in Feld Steven \& Basso Keith (eds.), Senses of Place, School of American Research Press, Sante Fe, New Mexico, pp. 91-135.

Feld Steven \& Brenneis Donald, 2004, "Doing Anthropology in Sound" in American Ethnologist, $n^{\circ} 31(4)$, pp. 461-474.

Feld Steven \& Boudreault-Fournier Alexandrine, 2019, « Relations sonores : entretien avec Steven Feld (University of New Mexico) » in Anthropologie et Sociétés, no 43(1), p. 195-210.

Feld Steven, Leonard Dennis \& Ra Richards Jeremiah, 2019, Voices of the Rainforest: A Day in the Life of Bosavi, Papua New Guinea, Audio $2^{\text {nd }}$ edition CD, BluRay 7.1 film with Concert and Ambient programs, and photographic book, VoxLox/Bosavi Peoples Fund, Santa Fe, NM. 
Gélard Marie-Luce, Gosselain Olivier P. \& Legrain Laurent, 2016, « La promenade sensorielle comme outil pédagogique » in Hermes, La revue, $\mathrm{n}^{\circ} 1$, p. 158-167.

Haeckel Ernst, 2012 [1866], Morphologie générale des organismes, Hachette Livre BNF, Paris, 632 p.

Ingold Tim, 2013, Marcher avec les dragons, Éditions du Seuil, Paris, 384 p.

Lussault Michel, 2020, Chroniques de géo'virale, École urbaine de Lyon et Éditions deux-cent-cinq (coll. À partir de l'Anthropocène), Lyon, 112 p.

Lussault Michel, Paquot Thierry \& Younès Chris (dir.), 2007, Habiter, le propre de l'humain. Villes, territoire et philosophie, La Découverte (coll. Armillaire), Paris, $384 \mathrm{p}$.

Maleval Véronique, Picker Marion \& Gabaude Florent, 2012, Géographie poétique et cartographie littéraire, Presses universitaires de Limoges et du Limousin, Limoges, 290 p.

Morizot Baptiste, 2020, « Politiser l'émerveillement et armer l'amour du vivant » in Socialter, hors-série Renouer avec le vivant, p. 6-9.

Privat Jean-Marie, 2019, «Oralité/Auralité » in Pratiques, nº 183-184, DOI : https://doi.org/10.4000/pratiques.6777.

Schafer R. Murray, 1977, The Soundscape: Our Sonic Environment and the Tuning of the World, Destiny Books, Rochester, Vermont, 301 p.

Sinaï Agnès, Carton Hugo, Servigne Pablo \& Stevens Raphaël, 2017, Petit Traité de résilience locale, éditions Charles Léopold Meyer, Paris, 104 p. 